\title{
An Experimental Research on Microseismic Monitoring Technology in Detecting the Contact Time of Badminton Amateurs' Lunge
}

\author{
Yang Yang \\ Department of PE \\ Beijing Jiaotong University \\ Beijing, China \\ yyang1@bjtu.edu.cn
}

\author{
Yingchun Cui† \\ Department of PE \\ Beijing Jiaotong University \\ Beijing, China \\ ychcui@bjtu.edu.cn
}

\author{
Wenfa Yan \\ Department of PE \\ Beijing Jiaotong University \\ Beijing, China \\ yanwenfa@bjtu.edu.cn
}




\section{ABSTRACT}

The fast and flexible characteristics of badminton determine the necessity of its lunge, and fast lunge will have an important impact on the results of the game in actual combat. In lunge evaluation, the contact time to the ground is a key indicator. This article selects two middle-aged male badminton amateurs with similar body shape and age, but different skill levels as the research objects to carry out comparative experiments. The microseismic equipment is used to record the floor vibration which is caused by the running of badminton amateurs in sports. The microseismic signals of lunge are processed and analyzed by MATLAB software. And we evaluate the pro and cons of various time to touch the ground from amateurs with different skill levels. The research found that 1 . Microseismic technology can detect the contact time to the ground. 2. High-level badminton amateurs have obvious advantages in the footwork contact time. Microseismic technology has a certain feasibility as a tool for evaluating the footwork contact time of badminton.

\section{KEYWORDS}

badminton, amateurs, lunge evaluation, contact time, microseismic, feasibility

\section{Introduction}

As early as 2009, the survey data of the Group Department of the State General Administration of Sports showed that badminton ranked second most popular sport among Chinese people, second only to brisk walking. As of 2016, the data still showed that badminton is the second most popular sport. According to statistics released by the Table Tennis and Badminton Center of the General Administration of Sports of China, there are 250 million people playing badminton in China. It can be said that badminton has a broad mass basis. Badminton also has the reputation of being the fastest racket sport in the world. This sport is a type with complex movements and strong technicality. With the increasing development of badminton, badminton not only is more abundant in content, but also the increasing difficulty of the action. The basic techniques of badminton can be roughly divided into two categories: technique on hand and the lunge. Athletes will make quick, flexible and changeable lunge conversions on the court according to the shuttle coming from the opponent, so that they can return the shuttle in the most reasonable way in time. If the athlete's lunge is poor and cannot be in the right place on time, it will affect the accuracy of the stroke, and even fail to receive the shuttle which resulting difficult use of the best shot technique. Even with the best stroke technique, it is difficult to use it. It shows the importance of lunge in badminton. Therefore, as an important part of badminton theory and technology, badminton lunge should attract the attention of coaches, athletes, amateurs and scientific researchers. The attributes of badminton lunge include contact time, step length, the force between the foot and the ground, and the angle of the joints. Among them, contact time is a critical indicator. From the moment a foot just touching the ground to completely leaving the ground, the time spent in this process is the contact time. The contact time has received extensive attention in the dynamic function research of various running sports. The average runner's contact time may be 300 milliseconds, and most amateur runner's contact time reaches 220-240 milliseconds, which is considered a good level. The elite runner's contact time can be controlled within 200 milliseconds, or even 150 (or less). Jamaica's runner Usain Bolt contact time reached a staggering 78 milliseconds.

The more common methods for the contact time research include mufti-parameter sensors placed on footwear, motion capture systems, pressure plates, high-speed camera systems, etc. The recording, analysis and evaluation of badminton lunge is an effective way to improve the badminton theory technical system, as well as the quality of training. The existing badminton lunge acquisition and recording methods mainly include wearable sensors and high-speed cameras. The Malaysian Kokum Weeratungai team used high-speed cameras to extract the athletes' frequent movement trajectory information on the badminton court [1]. The British Mark King team used a motion capture 
system including 18 cameras to record the threedimensional movement data of athletes, rackets and shuttles [2]. The Malaysian Azmin Rambely team used video analysis methods to analyze the contact time and take-off height selection of badminton players in the process of jumping and killing [3]. The Lin Hengwen team analyzed the characteristics of the use of asynchrony in badminton using video analysis methods [4]. King Zhelong, et al used wearable sensors to form a network to analyze the asynchrony in badminton [5]. Chen Qi, et al., of China used wireless sensor network to collect pace data to improve training effect [6]. In other sports, many scholars used wearable sensing devices and high-speed camera equipment to collect and evaluate athletes' sports data. Chinese Wang Yufan team reported the use of wearable sensing devices to assess the technical level of volleyball players [7]. J. Lee used a motion capture system composed of 16 inertial sensors to track whether the rider's movements were standard and established a database of motion characteristics for the rider. Analyze and instruct the movements of [8]; Basilio Pueo, et al discussed the motion capture application of high-speed camera equipment in sports events [9]. The Wing-Kai Lam of the Li-Ning Sports Science Research Center was based on a synchronized force measurement platform and motion analysis system, which deeply analyzed the lunge attributed of badminton players, and showed that the contact time of skilled badminton players was better than that of unskilled badminton players [10]

The wearable sensors or high-speed camera motion capture systems currently in use provide powerful assists for the analysis of the contact time and can clearly and accurately reflect the athlete's lunge attributes. But there are also some shortcomings for wearable sensors, no matter how light wearable sensors are, it will always interfere with the performance of athletes to a certain extent, and the sensors are only suitable for training and intrateam sports analysis. It is impossible for athletes to wear sensors during the match, and it is also impossible for the opponents to wear sensors and share data; The disadvantages of high-speed camera systems are high cost, inconvenience to carry, and are greatly restricted by practical factors such as size of the field and line of sight. In view of the above shortcomings, this paper proposes a method in detecting the contact time of badminton amateurs' lunge using Microseismic monitoring technology. This method has the advantages of high accuracy, convenient deployment, no impact on the line of sight, and will not affect badminton players.

\section{Research Objects and Methods}

\subsection{Research Objects}

Two middle-aged male badminton amateurs of similar body shape and age were selected as the research objects. Badminton amateur A, height $178 \mathrm{~cm}$, weight $75 \mathrm{~kg}$, 44 years old; and badminton amateur $B$, height $179 \mathrm{~cm}$, weight $76 \mathrm{~kg}$, 49 years old.

\subsection{Experimental Methods}

\section{Experimental Equipment}

The microseismic monitoring system used in this article is the Meridian Compact PH compact broadband microseismic all-in-one machine made in Canada, including a microseismic sensor and a collector. The physical map is shown in Figure 1. The microseismic sensor is easy to install, does not need to lock or center, and has a special small size and self-adaptive leveling system, which can significantly reduce the time and physical effort required for preparatory work and installation. 


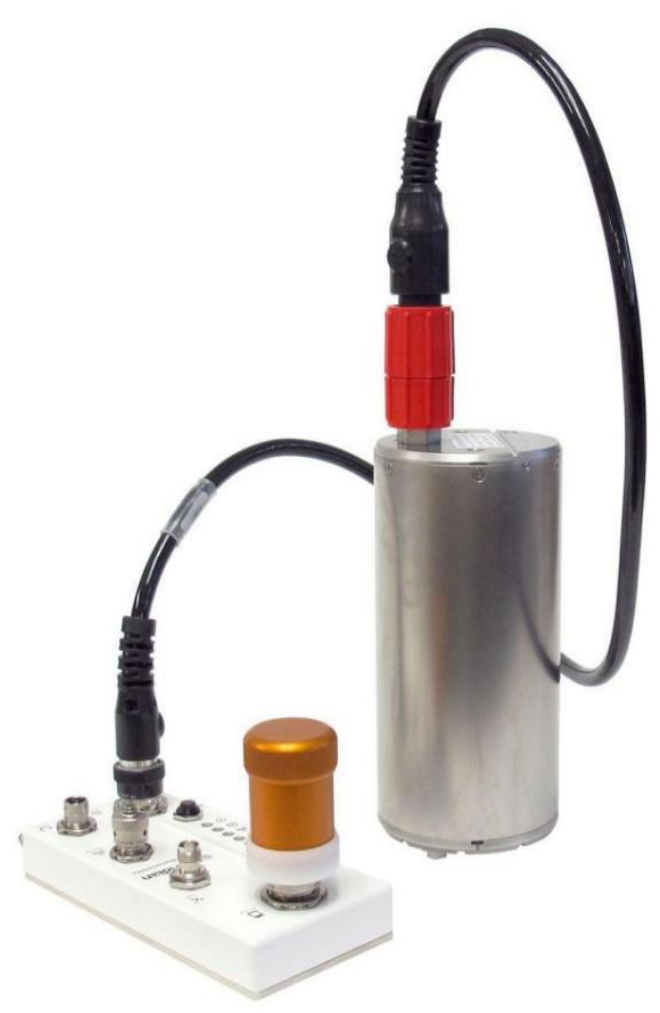

Figure 1: Microseismic system

The microseismic sensor used in this experiment has higher sensitivity and a larger range than the conventional microseismic sensor. Once the sensor is set up, it will begin to establish its own meta-data. The integrated design of the data acquisition and microseismic sensor unit can ensure the continuous and high-quality data.

\section{Experimental method}

In order to study the difference in the contact time of the amateurs, the experimental plan is as follows: An ordinary badminton court is used as a test site, and a fixed measuring point is set as shown in Figure 2. Badminton amateur A and badminton amateur B stand separately at position 17 , according to their own exercise habits, following the order of running to position 4 , position 5 , position 6 , position 18 , position 12 , position 11 , position 10 , and then return to position 17 each time.

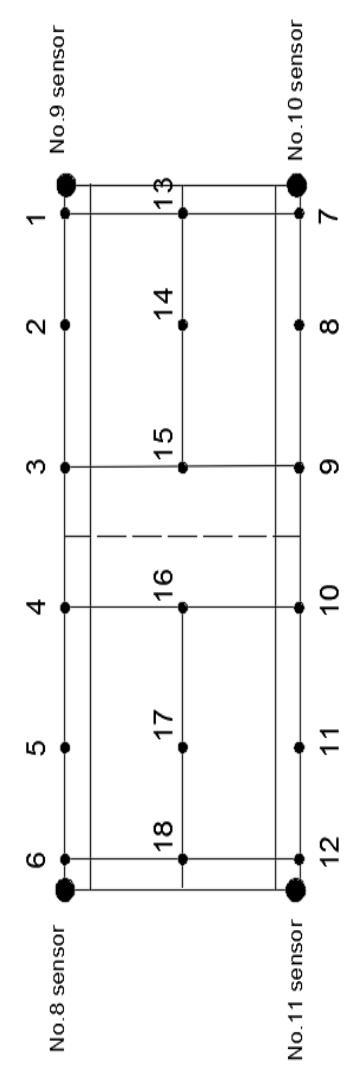

Figure 2: Schematic diagram of the actual measurement plan of the contact time

\subsection{Microseismic Data Analysis Method}

This microseismic data processing is mainly through MATLAB signal processing and visualization modules. The analysis method is based on the classic parameter analysis method of microseismic and acoustic emission signals [11], see Figure 3. Perform ring counts based on footwork, and extracted by the microseismic data of lunge. 


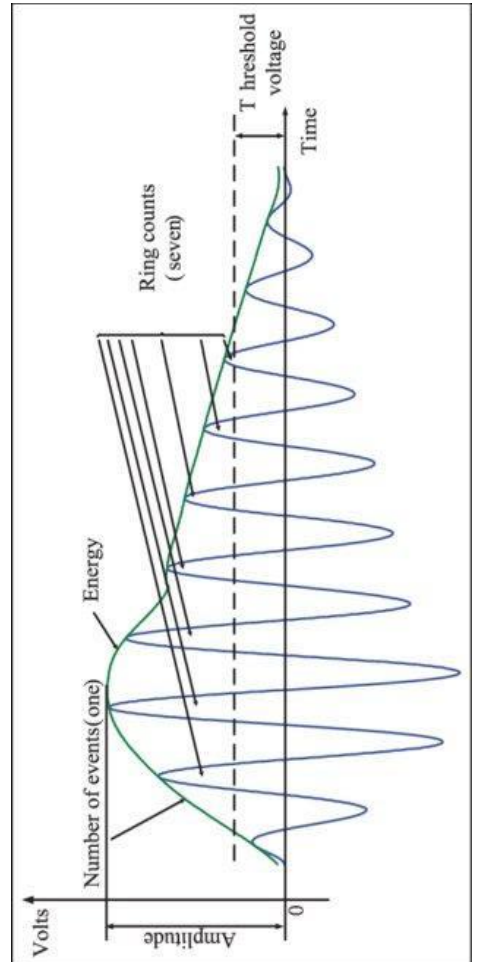

Figure 3: Schematic diagram of the basic method of microseismic signal analysis

The ring counts is the number of times that the microseismic signal waveform exceeds the set threshold. In general, the sampling rate is the same, and the ring counts of the microseismic waveform signal has a close positive correlation with the signal duration. If the lunge is dragging, the ring counts of the lunge vibration is high and the contact time is long. if the lunge is fast, the ring counts of the lunge vibration is low, and the contact time is short.

\section{Result}

The measured lunge vibration data of badminton amateur A and badminton amateur B are processed with MATLAB. The analysis shows that the average ring counts of badminton amateur B is $34.59 \%$ longer than that of badminton amateur $\mathrm{A}$.

Table 1 The lunge of two amateurs and the 6 measured contact time data

\begin{tabular}{|c|c|c|}
\hline No. & Amateur A & Amateur B \\
\hline 1 & 1357 & 2695 \\
\hline
\end{tabular}

\begin{tabular}{|c|c|c|}
\hline 2 & 1758 & 2636 \\
\hline 3 & 1101 & 2523 \\
\hline 4 & 2169 & 2333 \\
\hline 5 & 1658 & 2336 \\
\hline 6 & 2132 & 3032 \\
\hline Average & 1695.83 & 2592.5 \\
\hline
\end{tabular}

Comparing the ring counts of two badminton amateurs in each experiment, the ring counts of badminton amateur $\mathrm{A}$ is approximately twice that of badminton amateur $\mathrm{B}$. It is speculated that the lunge of badminton amateur $\mathrm{A}$ is relatively faster and has a short contact time, while B is more procrastinated and has a long contact time. If in the same game, because B has a longer contact time for each step, $B$ 's lunge time consumption is significantly higher than A under the same circumstances. Comparing the experimental results, the lunge level of badminton amateur $\mathrm{A}$ is higher than the lunge level of badminton amateur B, which is basically consistent with the results of daily training and competitions. It can basically be confirmed that the microseismic method is feasible to evaluate the badminton ground contact time.

\section{Discussion}

This article collects and analyzes the micro-seismic data of the lunge of two badminton amateurs. The results show that there is a significant difference in contact time for badminton amateurs of high and low levels, and the lunge of high-level amateurs is faster. This result is basically consistent with the results and empirical judgments of daily training and competitions. It can be considered that the microseismic method provides a new tool for the lunge evaluation research of badminton technical statistics, and we look forward to further research. 


\section{REFERENCES}

[1] Kokum Weeratungai, Anuja Dharmaratne, Khoo Boon How. (2018). Classification of Spatio-Temporal Data Represented as Multiset for Tactical Profiling in Badminton. 2nd International Conference on Image, Vision and Computing

[2] Mark King, Harley Towler, Romanda Dillon and Stuart McErlain-Naylor. (2020). A Correlational Analysis of Shuttlecock Speed Kinematic Determinants in the
Badminton Jump Smash. Appl. Sci. 2020, 10, 1248; doi:10.3390/app10041248

[3] Rambley A S, Abas WABW.(2008). Contact time and take-off speed relationship in determining height of jump in jumping badminton smash[C]//ISSNConference Proceedings Archive.

[4] Lin H W, Huang K S, Pan K M, et al.(2015). Biomechanical analysis of badminton different forward steps[C]//ISBS-Conference Proceedings Archive.

[5] Wang Z, Guo M, Zhao C.(2016). Badminton stroke recognition based on body sensor networks[]]. IEEE Transactions on Human-Machine Systems, 46(5): $769-775$.

[6] Qi C, Wei H, Shu-ming X, et al. (2012). Development of Wireless Sensor Network for Data Collection and lunge Training[]. Procedia Engineering, 34: 520-525.

[7] Yufan Wang, Yuliang Zhao, Rosa H. M. Chan, and Wen J. Li. (2018). Volleyball Skill Assessment using a Single Wearable Micro Inertial Measurement Unit at Wrist. IEEE Access. doi: 10.1109/ACCESS.2018.2792220

[8] J. Lee. (2014). A Three-Dimensional Motion Analysis of Horse Rider in Wireless Sensor Network Environments[]]. (IJACSA) International Journal of Advanced Computer Science and Applications: 2014, 5(11): 50-55

[9] Basilio Pueo. (2016). High speed cameras for motion analysis in sports science. JOURNAL OF HUMAN SPORT \& EXERCISE ISSN 1988-5202, doi:10.14198/jhse.2016.111.05

[10] Lam W-K, Lee K-K, Park S-K, Ryue J, Yoon S-H, Ryu J (2018) Understanding the impact loading characteristics of a badminton lunge among badminton players. PLoS ONE 13(10): e0205800

[11] Jiang K, Han L, Zhou Y. (2019). Quantitative evaluation of the impurity content of grease for low-speed heavy-duty bearing using an acoustic emission technique. Measurement and Control. 52(7-8):1159-1166. 